\title{
Performance Analysis of Deep Belief Neural Network for Brain Tumor Classification
}

\author{
${ }^{1}$ Sreenivas Eeshwaroju \& ${ }^{2}$ Praveena Jakula \\ ${ }^{1}$ Harman Connected Services, Novi, Michigan, USA. \\ ${ }^{2}$ Intel Corporation, USA. \\ ${ }^{* *}$ Corresponding Author: Sreenivas.Eeshwaroju@harman.com
}

Received: 30.08 .2020 , Revised: 20.11 .2020 , Accepted: 17.12 .2020 , Published: 22.12.2020

DOI:

10.53409/mnaa.jcsit20201305

Abstract: The brain tumors are by far the most severe and violent disease, contributing to the highest degree of a very low life expectancy. Therefore, recovery preparation is a crucial step in improving patient quality of life. In general, different imaging techniques such as computed tomography ( CT), magnetic resonance imaging ( MRI) and ultrasound imaging have been used to examine the tumor in the brain, lung, liver, breast, prostate ... etc. MRI images are especially used in this research to diagnose tumor within the brain with classification results. The massive amount of data produced by the MRI scan, therefore, destroys the manual classification of tumor vs. nontumor in a given period. However for a limited number of images, it is presented with some constraint that is precise quantitative measurements. Consequently, a trustworthy and automated classification scheme is important for preventing human death rates. The automatic classification of brain tumors is a very challenging task in broad spatial and structural heterogeneity of the surrounding brain tumor area. Automatic brain tumor identification is suggested in this research by the use of the classification with Deep Belief Network (DBN). Experimental results show that the DBN archive rate with low complexity seems to be $97 \%$ accurate compared to all other state of the art methods.

Keywords: Magnetic Resonance Imaging, Brain tumor and Deep Belief Network .

\section{INTRODUCTION}

he incidence of central nervous system (CNS) tumors in India ranges from 5 to 10 per 100,000 population with an increasing trend and accounts for $2 \%$ of malignancies [1]. Brain MRI image is mainly used to identify the process of modeling tumor and tumor progression. Such information is used mainly for procedures of tumor diagnosis and treatment. The MRI image contains more detail about the medical condition provided than the CT or ultrasound condition. The MRI image contains accurate brain structure and irregularity identification evidence in brain tissue.

In addition, from the time when it became possible to scan and bring medical imagesto the machine, Scholars provided unlike automated methods for brain tumor detection and typecataloging using brain MRI imaging. In comparison, Neural Networks ( NN) and Support Vector Machine ( SVM) have been the most widely used approaches for their successful implementation over the last few years[2].
Even so recently, models of Deep Learning ( DL) set a compelling trend in machine learning as the massive underground architecture can effectively represent complex relationships without needing a large number of nodes, such as in the superficial architectures. K-Nearest Neighbor (KNN), and Vector Machine Support (SVM). As a result, they evolved rapidly to become the state of the art in areas such as medical image processing, medical informatics and bioinformatics, apart from in health informatics.

This work proposes the DBN for the classification of brain tumors in MRI with this motivation. The proposed model to improve tumor and non-tumor detection rates with high accuracy. The primary contributions of this work are as follows:

- It is the first systematic method for the classification of MRI brain tumors using new Deep Neural Belief (DBN) networks

- Evaluation of the performance of the new framework demonstrating state-of-the- 
art output in contrast with current approaches.

The rest of the paper is organised as follows. Section 2 deals with the classification of MRI brain tumor. Section 3 describes the methods proposed for defining the MRI brain tumor classification with DBN. Section 4 discusses the experimental findings. Section 5 includes the conclusion and prospective work.

\section{RELATED WORK}

In a framework combined with a global probabilistic image model, local tissue intensity model and priors of the Markov Random Field[2] are introduced which may impede the automated methods of tissue classification in Brain IRM. A different method for the classification of MRI brain image is suggested by that of the integration of wavelet entropy-based web plots and probabilistic neural network. The two-stage classification process utilizes Spider Web Plots based on wavelets for the extraction of the characteristics and a probabilistic neural network for the classification[3].

The [4] fuzzy logic hybrid kernel has been created and applied for the automatic classification of four cancer types, including the meningioma, glioma, astrocytoma, and metastases called fuzzy logic hybrid kernel SVM, for the support of the Vector Machine.

In [5] a two techniques fusion is employed to identify the imperatives in brain tumor, namely the Tolerance Rugh Set (TRS) and the FireflyAlgorithm (FA). With [6] a CAD system can be connected to a SVM classification using a quadratic kernel function to support radiologists in their diagnosis procedures through a series of pre-processing, segmentation and extraction steps.

The self-organizing neural mapping network initiates [7] by training the features extracted from the discrete wavelet mixing wavelets and thus train the K-nearest neighbor in the filter factors and complete the testing process in two phases.

In [8], a brain tumor segmentation and classification method for MRI scans was proposed. The integrated characteristics are subsequently provided in five classes to the random forest classifier. DBN is being used to classify brain tumor images of MRI in this proposed system.

\section{PROPOSED METHODOLOGY}

This section presents the overall structure of the classification system for MRI brain tumors, and the architecture is shown in Figure 1. The analysis and modeling is made up ofthree stages, namely pre-processing, extraction of features, and classification. The training brain MRI image is provided as input to the system during the training process and is subjected to all of the above listed steps. A classifier model is selected DBN, where the extracted features are used to train the classifier along with the class labels. The DBN classifier now recognizes the features of the test image and will allocate a class label to the test image as either 'tumor affected' or 'tumor unaffected' with the information it has already acquired during the training process.

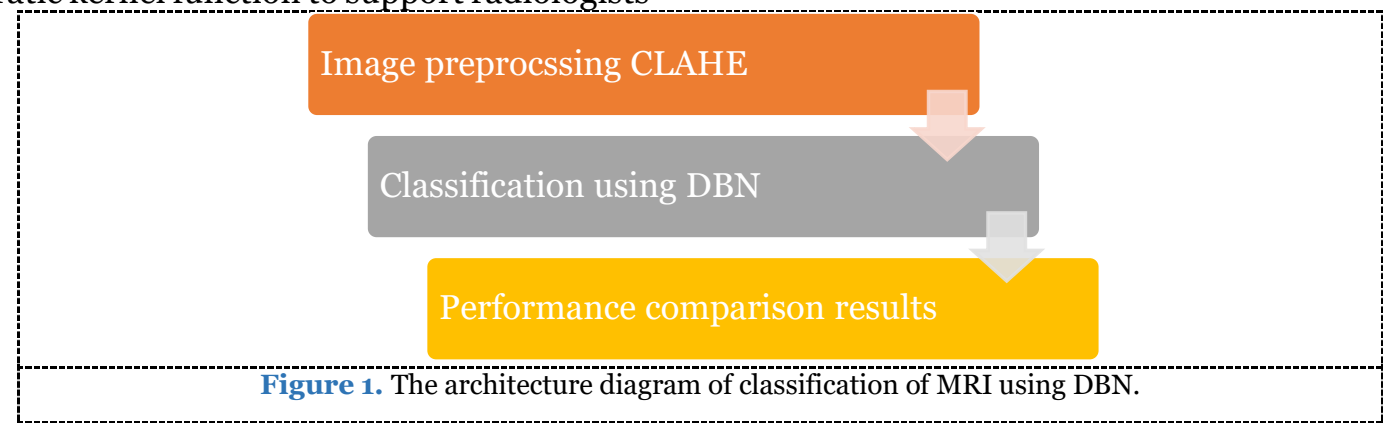

\subsection{Dataset Collection and Description}

The data collection of brain tumors obtained from [7] contains 30,064 T1-weighted contrastenhanced images. The dataset is compiled from 233 patients with three brain tumor types: meningioma (708 images), glioma (1426 images), and pituitary tumor (930 images). What file reserves a struct which comprises different image fields. The label (1 for meningioma, 2 for glioma, 3 for pituitary tumor), PID (patient ID), image info, tumor border, and tumor mask are included.

\subsection{Image Preprocessing using CLAHE}

The first phase in the method for classifying brain tumors in MRI is preprocessing. It preprocesses the input image in such a way that the output image shifts. For this work wiener filter Contrast Limited Adaptive Histogram Equalization (CLAHE) does not find the image in its entirety but separates it as tiles and improves the contrast of 
each tile while enhancing the contrast of the whole image. Used for reducing noise effects in the image. The output is taken as the input to the CLAHE after applying wiener filter which is used to enhance the contrast of the given input image. CLAHE algorithm is prearranged as follows:

Step 1: Read the input image.

Step 2: Put on Wiener filter to remove noise.

Step 3: Look after discover the number of frequencies for each pixel.

Step4: The transformation function is calculated using probability density of the input MRI brain image grayscale significance is utilized for amend all histograms, where $n$ remains the total number of pixels in the input MRI brain image and $n_{j}$ stands the input pixel number of grayscale value $j$.

Step 5: The grayscale values for the MRI-image are accustomed established on the effects of modified histograms besides bilinear interpolation actuality used modify the neighboring MRI-image.

Step 6: The perseverance of histogram equalization mapping is to deliver input MRI brain image intensity values in such a way as to deliver an essentially uniform distribution of the histogram from the resulting images. The histogram of MRI brain image with gray levels in the assortment $[0, L-1]$ is through discrete function $p\left(\right.$ gray $\left._{k}\right)=$ $\frac{n \times k}{n}$ somewhere gray $_{k}$ remains the $k$ th gray level, $n \times k$ stands the number of pixels in MRI brain image with the gray level, here $k=0,1,2, \ldots, L-1$. Mainly, $p\left(\right.$ gray $\left._{k}\right)$ provides an evaluation of the occurrence probability of gray level in MRI brain image.

Step 7: Display the enhanced MRI brain image.

\subsection{MRI brain tumor classification Using Deep Belief Networks}

Professor Geoffrey Hinton develops Deep Belief Networks (DBN) consisting of two distinct forms of neural networks-Belief Networks and Restricted Boltzmann Machines-to address the weakness of earlier neural networks. Here the emphasis was on the DBN-based improved Restricted Boltzmann Machines.

Boltzmann System is a recurrent stochastic neural network with binary stochastic units and undirected edges between units. Unfortunately, Boltzmann machine learning is inefficient, and has a problem of scalability. This resulted in the implementation of Restricted Boltzmann Machine (RBM)[10], which has one layer of hidden units and limits relations between hidden units. It allows for more effective learning algorithms[11]. The RBM structure is shown in figure 2 below.

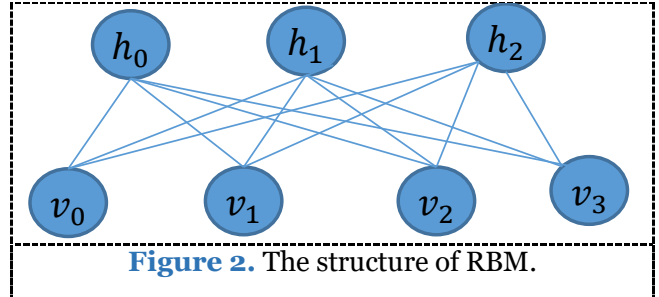

As Deep Belief Networks (DBN) name indicates, it As the name of Deep Belief Networks (DBN) indicates, it is networks of multilayer beliefs[12]. That layer is Restricted Boltzmann Machine and are stacked to create DBN. The first step of training DBN is to use the Gaussian Bernoulli RBM algorithm to learn a layer of features from the visible units 8 . Then the next step is to treat the activations of features previously trained as visible units and learn features in a second hidden layer.

Eventually, when learning for the final secret layer is reached the entire DBN is trained. The Gaussian Bernoulli RBM is used, since the input data of the deep architecture are real values. It is composed of a visible layer, a hidden layer and a layer of output. The energy relationship can be written in equation (1-3) and the conditional probability distribution of:

$$
\begin{aligned}
& \quad E(v, h \mid \theta)=\sum_{i=1}^{V} \frac{\left(v_{i}-a_{i}\right)^{2}}{2 \sigma_{i}^{2}}-\sum_{i=1}^{V} b_{j} h_{j}- \\
& \sum_{i=1}^{V} \sum_{j=1}^{H} \frac{v_{i}}{\sigma_{i}} h_{j} w_{i j} \\
& p\left(h_{i} \mid v ; \theta\right)=\delta\left(\sum_{i=1}^{V} w_{i j} v_{i}+b_{j}\right) \\
& p\left(v_{i} \mid h ; \theta\right)=N\left(\sigma_{i} \sum_{j=1}^{H} w_{i j} h_{j}+a_{i}, \sigma_{i}^{2}\right)
\end{aligned}
$$

where $\theta=(w, a, b), w_{i j}$ is the connection weight between the visible unit $v_{i}$ and hidden unit $h_{j}, a_{i}$ is the bias value of $v_{i}, b_{i}$ is the bias value of $h_{j}, \delta(x)$ can be logistic function and $N\left(\mu, \sigma_{i}^{2}\right)$ is the probability with the mean $\mu$ and variance $\sigma^{2}$. The flowchart of DBNs depth is given in Figure 3.

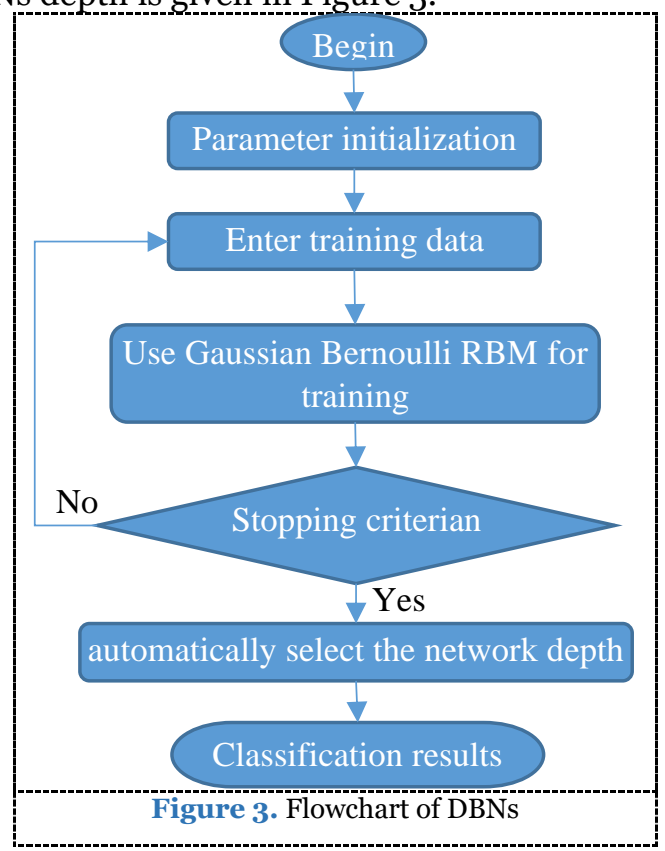




\section{EXPERIMENTAL RESULTS AND DISCUSSION}

The performance of the proposed DBN is evaluated in this section, and the performance results are compared with existing probabilistic neural network [3], Fuzzy Logic-Based Hybrid Kernel SVM [4] and KNN [7] schemes. The performance measurement is done in terms of precision, f-measurement, recall and accuracy.

Precision: It reflects the proportion of positive samples correctly classified as expected in equation (5):

$$
\text { Precision }=\frac{T P}{F P+T P}
$$

Recall: The recall of a classifier reflects the positive samples properly assigned to the total number of positive samples and is calculated as in equation (6):

$$
\text { Recall }=\frac{T P}{T P+F N}
$$

F-measure: this is also referred to as F 1-score, and as in equation (7) is the harmonic mean of precision and recall:

$$
F-\text { measure }=\frac{2 *(\text { Recall } * \text { Precision })}{(\text { Recall }+ \text { Precision })}
$$

Accuracy: This is one of the most frequently used performance classification measures and is defined as a ratio between the correctly classified samples and the total number of samples as in equation (8):

$$
\text { Accuracy }=\frac{T P+T N}{T P+T N+F P+F N}
$$

Where true positive (TP) samples are properly classified as no tumor, false positive (FP) samples are incorrectly classified as tumor, True negative (TN) samples are properly classified as tumor, and false negatives (FN) are incorrectly classified as tumor.

\subsection{Precision Rate comparison}

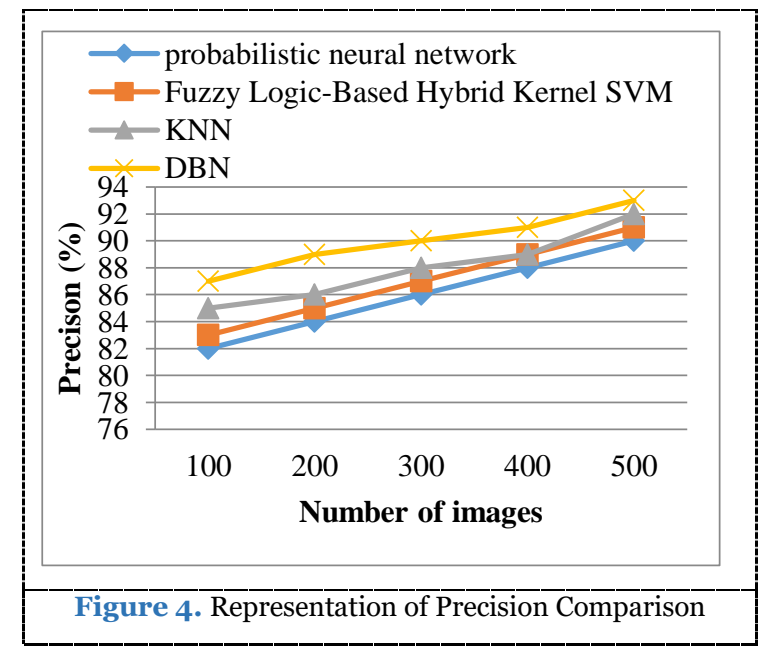

From the above Figure 4, the graph shows how accurate the number of images in the specified datasets is compared. These methods are implemented as probabilistic neural network, Fuzzy Logic-Based Hybrid Kernel SVM, KNN and DBN. When the number of records increases according to the precision value, from this graph, it is learned that the proposed DBN offers 93\% higher precision than previous methods that yield better results in the classification of MRI brain tumor due to Gaussian Bernoulli RBM. The numerical results of Precision Comparison is shown in Table 1.

Table 1. The numerical results of Precision Comparison

\begin{tabular}{ccccc}
\hline $\begin{array}{c}\text { No.of } \\
\text { images }\end{array}$ & $\begin{array}{c}\text { probabilistic } \\
\text { neural } \\
\text { network }\end{array}$ & $\begin{array}{c}\text { Fuzzy } \\
\text { Logic- } \\
\text { Based } \\
\text { Hybrid } \\
\text { Kernel } \\
\text { SVM }\end{array}$ & KNN & DBN \\
\hline 100 & 82 & 83 & 85 & 87 \\
\hline 200 & 84 & 85 & 86 & 89 \\
\hline 300 & 86 & 87 & 88 & 90 \\
\hline 400 & 88 & 89 & 89 & 91 \\
\hline 500 & 90 & 91 & 92 & 93 \\
\hline
\end{tabular}

\subsection{Recall comparison}

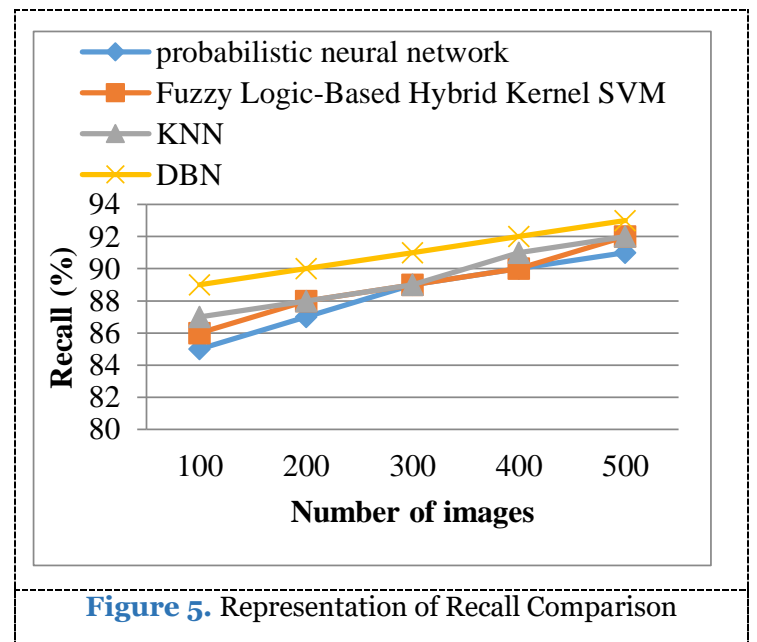

From the above Figure 5, the graph illustrates the recall relation for the number of images in the listed datasets. These methods are implemented as Fuzzy Logic-Based Hybrid Kernel SVM, KNN and DBN. Increasing the number of images often increases the correct value for the recall. Through this graph, it is discovered that the current DBN offers recall 93\% higher than previous methods. The explanation for this is that the DBN extracts the features directly, which will enhance the detection and classification of brain tumor. The numerical results of Recall Comparison is shown in Table 2. 
Table 2. The numerical results of Recall Comparison

\begin{tabular}{ccccc}
\hline $\begin{array}{c}\text { No.of } \\
\text { images }\end{array}$ & $\begin{array}{c}\text { probabilistic } \\
\text { neural } \\
\text { network }\end{array}$ & $\begin{array}{c}\text { Fuzzy } \\
\text { Logic- } \\
\text { Based } \\
\text { Hybrid } \\
\text { Kernel } \\
\text { SVM }\end{array}$ & KNN & DBN \\
\hline 100 & 85 & 86 & 87 & 89 \\
\hline 200 & 87 & 88 & 88 & 90 \\
\hline 300 & 89 & 89 & 89 & 91 \\
\hline 400 & 90 & 90 & 91 & 92 \\
\hline 500 & 91 & 92 & 92 & 93 \\
\hline
\end{tabular}

\subsection{F-measure Rate comparison}

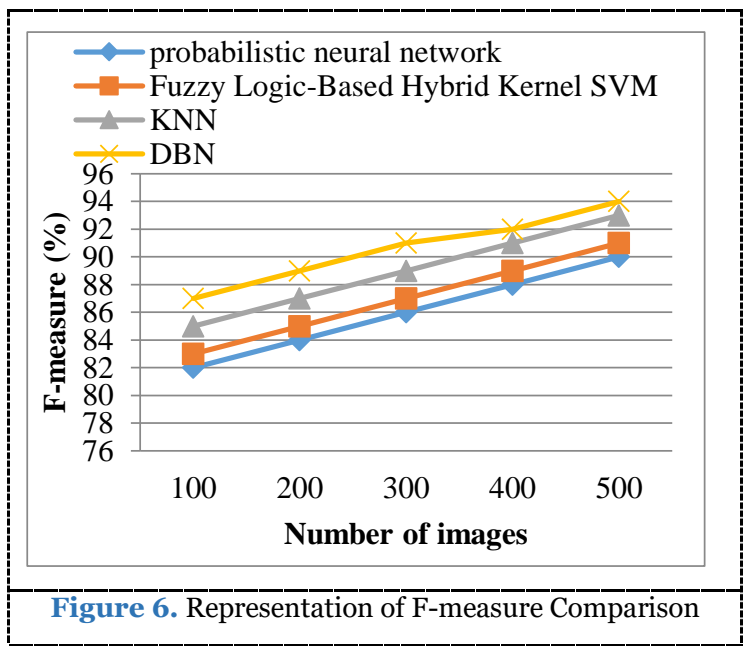

From the above Figure 6, the graph explains the fmeasure relation for the number of images in the given datasets. These methods are implemented as Fuzzy Logic-Based Hybrid Kernel SVM, KNN and DBN. When the number of data is increased, and the f-measure value is increased accordingly. From this graph it is learned that the proposed DBN offers 94\% higher f-measurement than previous methods. Therefore the proposed DBN algorithm is stronger than the current algorithms in terms of better performance of classifying MRI brain tumor. The numerical results of F-measure Comparison is shown in Table 3.

Table 3. The numerical results of F-measure Comparison

\begin{tabular}{ccccc}
\hline No.of & $\begin{array}{c}\text { probabilistic } \\
\text { neural } \\
\text { network }\end{array}$ & $\begin{array}{c}\text { Fuzzy } \\
\text { Logic- } \\
\text { Based } \\
\text { Hybrid } \\
\text { Kernel } \\
\text { SVM }\end{array}$ & KNN & DBN \\
\hline 100 & 82 & 83 & 85 & 87 \\
\hline 200 & 84 & 85 & 87 & 89 \\
\hline 300 & 86 & 87 & 89 & 91 \\
\hline 400 & 88 & 89 & 91 & 92 \\
\hline 500 & 90 & 91 & 93 & 94 \\
\hline
\end{tabular}

\subsection{Accuracy comparison}

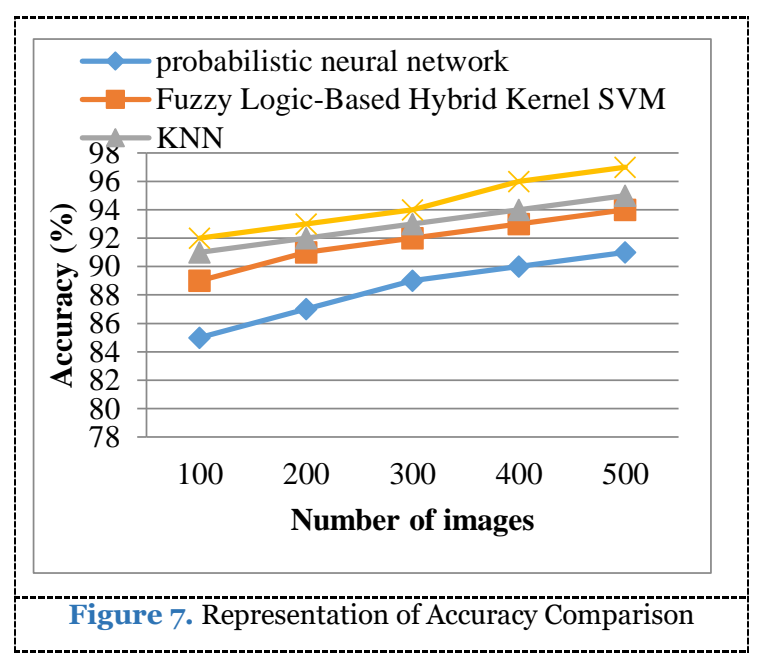

From the above Figure 7, the diagram illustrates the processing time relation for the number of images in the specified datasets. These methods are implemented as Fuzzy Logic-Based Hybrid Kernel SVM, KNN and DBN. From this graph, it is known that the proposed DBN algorithm is higher than the existing algorithms with a high precision rate of $97 \%$ in terms of better template matching results. This is due to the automatic extraction of the function in the DBN algorithm, which increases the MRI brain tumor classification results. The numerical results of Accuracy Comparison is shown in Table 4.

Table 4. The numerical results of Accuracy Comparison

\begin{tabular}{ccccc}
\hline No.of & $\begin{array}{c}\text { probabilistic } \\
\text { neural } \\
\text { network }\end{array}$ & $\begin{array}{c}\text { Fuzzy } \\
\text { Logic- } \\
\text { Based } \\
\text { Hybrid } \\
\text { Kernel } \\
\text { SVM }\end{array}$ & KNN & DBN \\
\hline 100 & 85 & 89 & 91 & 92 \\
\hline 200 & 87 & 91 & 92 & 93 \\
\hline 300 & 89 & 92 & 93 & 94 \\
\hline 400 & 90 & 93 & 94 & 96 \\
\hline 500 & 91 & 94 & 95 & 97 \\
\hline
\end{tabular}

\section{CONCLUSION AND FUTURE} WORK

This research indicates that DBN algorithms are used to extract features for the classification of MRI braiun tumors with a high precision rate of $97 \%$. The machine will considerably divide the tumor into three levels; meningioma, glioma, and pituitary tumor using contrast-enhanced brain MR images of $\mathrm{T} 1$ weight. Including more brain $\mathrm{MR}$ images with different weights and with various contrast enhancement techniques to allow the architecture to be potentially more versatile and reliable for larger image databases will further increase this architectural grading performance. The proposed model, nevertheless, still poses shortcomings such as long calculation time. The next research material 
will be how to refine the algorithm and shorten the run-time.

\section{REFERENCES}

[1]. Nair M, Varghese C, Swaminathan R. Cancer: Current Scenario, Intervention Strategies and Projections for 2015. NCMH Background Papers; 2015.

[2]. Tohka, J., Dinov, I. D., Shattuck, D. W., \& Toga, A. W. (2010). Brain MRI tissue classification based on local Markov random fields. Magnetic resonance imaging, 28(4), 557-573.

[3]. Saritha, M., Joseph, K. P., \& Mathew, A. T. (2013). Classification of MRI brain images using combined wavelet entropy based spider web plots and probabilistic neural network. Pattern Recognition Letters, 34(16), 2151-2156.

[4]. Jayachandran, A., \& Sundararaj, G. K. (2015). Abnormality segmentation and classification of multi-class brain tumor in MR images using fuzzy logic-based hybrid kernel SVM. International Journal of Fuzzy Systems, 17(3), 434443 .

[5]. Jothi, G. (2016). Hybrid Tolerance Rough Set-Firefly based supervised feature selection for MRI brain tumor image classification. Applied Soft Computing, 46, 639-651.

[6]. Natteshan, N. V. S., \& Jothi, J. A. A. (2015). Automatic classification of brain mri images using svm and neural network classifiers. In Advances in intelligent informatics (pp. 19-30). Springer, Cham.

[7]. Anitha, V., \& Murugavalli, S. J. I. C. V. (2016). Brain tumour classification using two-tier classifier with adaptive segmentation technique. IET computer vision, 1O(1), 9-17.

[8]. Usman, K., \& Rajpoot, K. (2017). Brain tumor classification from multi-modality MRI using wavelets and machine learning. Pattern Analysis and Applications, 20(3), 871881.

[9]. J. Cheng, 'brain tumor dataset', 2017. [Online]. Available: https://figshare.com/articles/brain_tumor_dataset/15124 27 ,

[10]. Hinton, G. E. (2012). A practical guide to training restricted Boltzmann machines. In Neural networks: Tricks of the trade (pp. 599-619). Springer, Berlin, Heidelberg.

[11]. Hinton, G., Srivastava, N., \& Swersky, K. (2012). Neural networks for machine learning. Coursera, video lectures, 264(1).

[12]. Arel, I., Rose, D. C., \& Karnowski, T. P. (2010). Deep machine learning-a new frontier in artificial intelligence research [research frontier]. IEEE computational intelligence magazine, 5(4), 13-18. 